\title{
TPX2 silencing mediated by joint action of microvesicles and ultrasonic radiation inhibits the migration and invasion of $\mathrm{SKOV} 3$ cells
}

\author{
DONG HUANG, JIANMIN CHEN, CUIYU YANG and MINZHEN WANG \\ Department of Obstetrics and Gynecology, Sir Run Run Shaw Hospital, School of Medicine, \\ Zhejiang University, Hangzhou, Zhejiang 310016, P.R. China
}

Received March 31, 2017; Accepted November 13, 2017

DOI: $10.3892 / \mathrm{mmr} .2018 .8810$

\begin{abstract}
Ovarian cancer, with its high morbidity, has one of the highest mortality rates among gynecological malignant tumors. Overexpression of targeting protein for Xklp2 (TPX2) has been identified in numerous malignant tumors. The present study sought to determine whether TPX2 silencing inhibited the growth and metastasis of ovarian cancer cells, and whether microvesicles- and ultrasonic radiation-mediated small interfering (si)RNA-TPX2 transfection may improve the therapeutic effect. The SKOV3 cell line, derived from papillary serous cytadenocarcinoma of the human ovary, was selected as a cell model. Cells were divided into five groups: Control, siRNA-TPX2, siRNA-TPX2 + microvesicle (M), siRNA-TPX2 + ultrasonic irradiation (UI), and siRNA-TPX2 + M + UI. Cell viability was evaluated under the aforementioned conditions via the Cell Counting kit 8 (CCK8) assay. Cell migration and invasion were detected using Transwell assays. The expression levels of associated genes, including epithelial cadherin (E-cadherin), metalloproteinase inhibitor 2 (TIMP-2), metastasis associated 1 (MTA1) and matrix metallopeptidase 2 (MMP2), were analyzed using reverse transcription-quantitative polymerase chain reaction analysis and western blotting. MMP2 activity was determined using a gelatin zymography assay. The results suggested that TPX2 serves an important role in the development of SKOV3 cells; it is additionally able to inhibit cell migration and invasion by upregulating E-cadherin and TIMP2, downregulating MMP2 and MTA1, and inhibiting the phosphorylation of p38 and c-Jun N-terminal kinase. The inhibitory effect of siRNA-TPX2 on SKOV3 cellular metastasis in the presence
\end{abstract}

Correspondence to: Dr Minzhen Wang, Department of Obstetrics and Gynecology, Sir Run Run Shaw Hospital, School of Medicine, Zhejiang University, 3 East Qingchun Road, Hangzhou, Zhejiang 310016, P.R. China

E-mail: 3197003@zju.edu.cn

Key words: gene silencing, ovarian cancer, microvesicles, ultrasonic irradiation, gene therapy, metastasis of microvesicles and ultrasonic radiation was observed to be improved compared with the control. It is proposed that the combination of microvesicles and ultrasonic radiation with TPX2 silencing has the potential to be an effective gene therapy against ovarian cancer.

\section{Introduction}

As one of the three predominant malignant tumors of the female reproductive system, ovarian cancer is difficult to diagnose at its onset. It has been reported that $>70 \%$ of patients with ovarian cancer have already reached a median or advanced phase of the disease when they consult a doctor (1). The mortality rate of ovarian cancer ranks second among all cancers of the female reproductive system (2). Therapy for ovarian cancer is based on surgical treatment supplemented by chemotherapy. Although chemotherapy may effectively combat cancer in the early stages of treatment, the majority of patients eventually experience cancer recurrence in the postoperative stage, and thus the 5-year survival rate for ovarian cancer is only $44 \%$ (3). Therefore, it is important to investigate novel therapeutic methods that may help improve the quality of treatment and the survival rate of patients with ovarian cancer.

With the recent advances in biomedical research, biotherapies, including gene therapy and immunotherapy, have attracted attention due to their great potential compared with current treatment strategies, including surgical treatment, radiotherapy and chemotherapy. However, being a novel therapeutic method, gene therapy also faces problems. Viral genetic transporters exhibit a high efficiency but high toxicity to cells, whereas non-viral vectors are comparatively safe but inefficient (4). With the development of ultrasound and associated imaging technology, ultrasound microvesicles as contrast agents have helped make notable progress in gene therapy. When microvesicles are applied as contrast agents, they may function as genetic vectors, following the principles of cavitation and sonoporation; this improves gene transfection in tissues or cells and may achieve the goal of effective cancer treatment (4-6).

Targeting protein for Xklp2 (TPX2) is a microtubule-associated protein (7). The expression of TPX2 is 
influenced by the cell cycle. The gene product appears during the G1-S stage and disappears following the completion of mitosis. Possessing a key role in the regulation of mitosis, TPX2 controls microtubule assembly and spindle stability in cooperation with Aurora-A kinase and Eg5 kinesin $(8,9)$. Furthermore, it serves a role in the formation of spindle apparatus and in chromosome segregation $(9,10)$. A number of research studies have provided evidence that TPX2 is overexpressed in numerous types of tumors, including lung, hepatic, colon, pancreatic and salivary gland cancer, which suggests a probable association of TPX2 with oncogenesis, or at least with certain associated malignancies (11-15). Overexpression of TPX2 results in the amplification of centrosomes and in DNA polyploidy (16). During interphase, TPX2 is preferentially located in the nucleus (9). Recently, TPX2 expression has been regarded as a marker for the diagnosis and prognosis of malignancies in a number of types of cancer $(9,11,12,17)$.

The present study explored the effects of TPX2 silencing in combination with two other treatments, microvesicles and/or ultrasonic radiation, on the ovarian cancer cell line SKOV3, to investigate the potential of this silencing phenomenon as an inhibiter of migration and invasion of ovarian cancer cells.

\section{Materials and methods}

Cells and recombinant plasmid. The cell line SKOV3 was obtained from the Cell Bank Type Culture Collection of Chinese Academy of Sciences (Shanghai, China) and cultured in Dulbecco's modified Eagle's medium (DMEM; Thermo Fisher Scientific, Inc., Waltham, MA, USA) contained with $10 \%$ fetal calf serum (Beijing Transgen Biotech Co., Ltd., Beijing, China) at $37^{\circ} \mathrm{C}$ in a $5 \% \mathrm{CO}_{2}$ incubator. The medium was changed every 2 days. Cell passage cultivation was commenced when cells grew to $90 \%$ confluence. Recombinant plasmids of small interfering (si)RNA-TPX2 were provided by Shanghai Sangong Pharmaceutical Co., Ltd. (Shanghai, China). The sequences of siRNA-TPX2 were 5'-GGAUGA UAUUAACCUGUUATT-3' and 5'-UAACAGGUUAAUAUC CUCCTT-3'.

Grouping and treatment. The SKOV3 cells were randomly divided into 5groups: Control, siRNA-TPX2, siRNA-TPX2 + microvesicles (M), siRNA-TPX2 + ultrasonic irradiation (UI), and siRNA-TPX $2+\mathrm{M}+\mathrm{UI}$. The microvesicles used in the present study were purchased from Cold Spring Biotech Corp. (Tapei, Taiwan). Except for the control, cells in all other groups were transfected with a recombinant plasmid, siRNA-TPX2, by different means, including pure transfection and transfection assisted with microvesicles and/or ultrasonic irradiation. Cells in the siRNA-TPX2 group were transfected with plasmids using Lipofectamine ${ }^{\circledR} 2000$ reagent (Thermo Fisher Scientific, Inc.), according to the manufacturer's protocol. Cells in the siRNA-TPX2 + M group were transfected with $4 \mu \mathrm{g}$ plasmid and $200 \mu \mathrm{l}$ microvesicles $(10 \%)$. In the siRNA-TPX2 + UI group, cells were transfected with $4 \mu \mathrm{g}$ plasmid, followed by treatment with UI at a frequency of $2.0 \mathrm{MHz}$ and mechanical index of 0.28 for $30 \mathrm{sec}$. In the siRNA-TPX $2+M+$ UI group, cells were transfected with a mixture of $4 \mu \mathrm{g}$ plasmid and $200 \mu \mathrm{l}$ microvesicles (10\%), followed by treatment with ultrasonic radiation with the same parameters as above for $30 \mathrm{sec}$. Following transfection, cells were incubated in $5 \% \mathrm{CO}_{2}$ at $37^{\circ} \mathrm{C}$ for $48 \mathrm{~h}$ prior to subsequent experiments.

Detection of cell viability. Cell viability in each group and the control was detected using a Cell Counting kit 8 assay (CCK8; Beyotime Institute of Biotechnology, Haimen, China). Cells $\left(3 \times 10^{4}\right.$ cells $\left./ \mathrm{ml}\right)$ were seeded into 96 -well plates with $100 \mu \mathrm{l}$ added per well; the plates were incubated at $37^{\circ} \mathrm{C}$ in the presence of $5 \% \mathrm{CO}_{2}$ for $4 \mathrm{~h}$. Cells were then supplemented with $10 \mu \mathrm{lCCK}$ reagent in each well, then placed in an incubator with $5 \% \mathrm{CO}_{2}$ at $37^{\circ} \mathrm{C}$ for $3 \mathrm{~h}$. The optical density of cells from each group was measured at $450 \mathrm{~nm}$ using a microplate reader (Bio-Rad Laboratories, Inc., Hercules, CA, USA).

Detection of cell migration and invasion. Transwell inserts (Corning Incorporated, Corning, NY, USA) were used to detect cell migration and invasion. For the migration assay, cells $\left(5 \times 10^{5}\right.$ cells $\left./ \mathrm{ml}\right)$ cultured in serum-free medium from each group were added to the upper chamber, and fetal bovine serum (Beijing Transgen Biotech Co., Ltd.) containing DMEM (Thermo Fisher Scientific, Inc.) was added to the lower chamber. All the cells were left to migrate for $24 \mathrm{~h}$. The migrated cells were fixed in $95 \%$ alcohol, stained with $0.1 \%$ hexamethyl pararosaniline (Beyotime Institute of Biotechnology, Haimen, China) for $20 \mathrm{~min}$ at room temperature, and counted under an upright metallurgical microscope (magnification, x100; Leica Microsystems GmbH, Wetzlar, Germany). The experiment was repeated 3 times for each group. For invasion detection, upper chambers of Transwell were specifically coated with Matrigel. The rates of cell migration and invasion in all groups were calculated.

Reverse transcription-quantitative polymerase chain reaction $(R T-q P C R)$. Cells were seeded into 6-well plates at a density of $2 \times 10^{6}$ cells/well. Total RNA was extracted using TRIzol (Thermo Fisher Scientific, Inc.), according to the manufacturer's protocols. The concentration of extracted RNA was read using a UV spectrophotometer (Thermo Fisher Scientific, Inc.). cDNA was synthesized using: A reverse transcriptase, a poly (A) polymerase and tagged oligo (dT) primers in a $20 \mu 1$ reaction volume for RT-qPCR (miScript; Qiagen, Inc., Valencia, CA, USA) according to the manufacturer's protocol. Each RT-qPCR contained $2 \mu 1$ of cDNA, $400 \mathrm{nM}$ of each sense and antisense primer, and 2x SYBR-Green PCR Master Mix (Takara Bio, Inc., Otsu, Japan). The reaction mixture was incubated at $37^{\circ} \mathrm{C}$ for $60 \mathrm{~min}, 85^{\circ} \mathrm{C}$ for $5 \mathrm{~min}$ and $4^{\circ} \mathrm{C}$ for $5 \mathrm{~min}$. The amplification assay was performed using ABI 7500 (Applied Biosystems; Thermo Fisher Scientific, Inc.). PCR was carried out by activating the DNA polymerase at $95^{\circ} \mathrm{C}$ for $10 \mathrm{~min}$, followed by 40 cycles of two-step PCR $\left(95^{\circ} \mathrm{C}\right.$ for $15 \mathrm{sec}$ and $60^{\circ} \mathrm{C}$ for $\left.45 \mathrm{sec}\right)$. GAPDH was used as the internal control to monitor the efficiency of RT-qPCR. All primers in the present study were designed by Shanghai Sangon Biotech Co., Ltd. (Shanghai, China). The specific primer sequences for each gene were as follows: 5'TCACAGCTCACTGCAGCC TT3' and 5'CTCTGGGAGGCCAAGATGGG3' for epithelial cadherin (E-cadherin; product, 196 bp); 5'GCCAAGTTC TTCGCCTGCAT3' and 5'AGCTGGACCAGTCGAAAC CC3' for metalloproteinase inhibitor 2 (TIMP-2; product, 
$168 \mathrm{bp})$; 5'CGAGACCGAGTCGCTCAAGT3' and 5'GCCATC CTCCTCGCCTTCTT3' for metastasis associated 1 (MTA1; product, $169 \mathrm{bp}$ ); 5'AGGACTACGACCGCGACAAG3' and 5'TGTGGTCGCACACCACATCT3' for matrix metallopeptidase 2 (MMP2; product, $173 \mathrm{bp}$ ) and 5'CGGGAAACTGTG GCGTGATG3' and 5'ATGACCTTGCCCACAGCCTT3' for GAPDH (product, $87 \mathrm{bp}$ ). Each reaction was run in triplicate. The $2^{-\Delta \Delta \mathrm{Cq}}$ method was performed for the quantification of gene expression data (18).

Gelatin zymography assay. Cells were centrifuged for $10 \mathrm{~min}$ at $310 \mathrm{x} \mathrm{g}$ at $4^{\circ} \mathrm{C}$ to remove suspended cells from all treatments. The supernatant from each was then resolved via $8 \%$ SDS-PAGE containing $1 \%$ gelatin. The gel was incubated in a renaturing buffer $(2.5 \%$ Triton $\mathrm{X} 100)$ for $30 \mathrm{~min}$ at room temperature, followed by incubation for a further $30 \mathrm{~min}$ in a developing buffer (Tris base, $50 \mathrm{mM}$; Tris $\mathrm{HCl}, 0.2 \mathrm{M}$; $\mathrm{NaCl}, 0.2 \mathrm{M} ; \mathrm{CaCl}_{2}, 5 \mathrm{mM}$; and $\mathrm{Brij}-35,0.02 \%$ ) with gentle agitation. The gel was subsequently incubated in developing buffer at $37^{\circ} \mathrm{C}$ overnight and stained with Coomassie R-250 (Sigma-Aldrich; Merck KGaA, Darmstadt, Germany) for $>2.5 \mathrm{~h}$ with gentle agitation at room temperature. The samples were viewed using the LI-COR Odyssey ${ }^{\circledR} \mathrm{CLx}$ infrared imaging system (LI-COR Biosciences, Lincoln, NE, USA) and analyzed using ImageQuant ${ }^{\mathrm{TM}}$ TL (version number 7.0; GE Healthcare, Chicago, IL, USA).

Western blot analysis. Cells were seeded into 6-well plates at a density of $2 \times 10^{6}$ cells/well. Cells were harvested and washed twice with phosphate buffer solution and lysed in ice-cold radioimmunoprecipitation assay buffer (Beyotime Institute of Biotechnology) with freshly mixed $0.01 \%$ protease inhibitor phenylmethane sulfonyl fluoride (Beyotime Institute of Biotechnology). The mixture was then incubated for $30 \mathrm{~min}$ on ice. Cell lysate was centrifuged at $10,000 \mathrm{xg}$ for $5 \mathrm{~min}$ at $4^{\circ} \mathrm{C}$. The supernatants containing 20-30 $\mu \mathrm{g}$ protein were collected, and protein concentration was determined using a BCA protein assay kit (Thermo Fisher Scientific, Inc.). Samples were then subjected to $10 \%$ SDS-PAGE, and transferred onto a nitrocellulose membrane (Merck \& Co., Inc., Kenilworth, NJ, USA). The membranes were blocked using $5 \%$ non-fat milk in Tris buffered saline containing $0.5 \%$ Tween-20 for $1 \mathrm{~h}$ at room temperature. Membranes were incubated with the following primary specific antibodies at $4{ }^{\circ} \mathrm{C}$ for $6 \mathrm{~h}$ and then at room temperature for $4 \mathrm{~h}$ : Anti-E-cadherin (cat. no. ab76055; 1:1,000), anti-TIMP-2 (cat. no. ab1828; 1:1,000), anti-MTA1 (cat. no. ab71153; 1:2,000), anti-MMP2 (cat. no. ab92536; 1:1,000), anti-phosphorylated-P-38 (phosphorylated Y182; cat. no. ab47363; 1:800); anti-P-38 (cat. no. ab170099; 1:1,000); anti-phosphorylated-JNK1 + JNK2 + JNK3 (phosphorylated T183 + T183 + T221; cat. no. ab179461; 1:1,000); anti-JNK1 + JNK2 + JNK3 (cat. no. ab8245; 1:1,000) and anti-GAPDH (cat. no. ab8245; 1:2,000; all Abcam, Cambridge, MA, USA). The membranes were then incubated with the following secondary antibodies at room temperature for $1 \mathrm{~h}$ : Goat anti-mouse IgG H\&L (cat. no. ab6789; 1:2,000), goat anti-rabbit IgG H\&L (cat. no. ab6721; 1:2,000) and donkey anti-goat IgG H\&L (cat. no. ab6885; 1:2,000; all Abcam). Blots were visualized via enhanced chemiluminescence (Thermo Fisher Scientific, Inc.). Densitometry of the bands was determined using ImageJ software (version 1.48; National Institutes of Health, Bethesda, MD, USA).

Statistical analysis. All statistical analyses were performed using SigmaStat 2.0 for Windows (Systat Software Inc., San Jose, CA, USA). Data were expressed as the mean \pm standard deviation. Comparisons of multiple treatment groups to controls were performed using one-way analysis of variance followed by Tukey and Bonferroni post-hoc tests. Differences among groups were evaluated through Student's t-test. $\mathrm{P}<0.05$ was considered to indicate a statistically significant difference.

\section{Results}

Characterization of microvesicles. Microvesicles added to the siRNA-TPX2 + M and siRNA-TPX2 + M + UI groups were clearly identified via an upright metallurgical microscope (magnification, $\mathrm{x} 400$ ). The grain size of these microvesicles was $1,071 \pm 469.04 \mu \mathrm{m}$. Their average electric potential was $11.35 \pm 2.11 \mu \mathrm{A}$ (Fig. 1A).

Inhibition of TPX 2 expression. The amount of TPX2 protein in each group and the control was detected by western blotting. This additionally provided information on the transfection efficiency of the siRNA-TPX2 plasmid under different conditions. In the siRNA-TPX2 group, no significant decrease in TPX2 protein levels was observed compared with the control group. In the siRNA-TPX2 + M and siRNA-TPX2 + UI groups, TPX2 was less expressed compared with the siRNA-TPX2 group $(\mathrm{P}<0.05$ and $\mathrm{P}<0.01$, respectively). The combination of ultrasonic radiation and microvesicles significantly inhibited the expression of TPX2 to one-fifth of the expression in control SKOV3 cells ( $\mathrm{P}<0.01$; Fig. 1B).

Cell viability was negatively affected by TPX2 silencing. Cell viability in the siRNA-TPX2, siRNA-TPX $2+\mathrm{M}$, siRNA-TPX2 + UI, and siRNA-TPX2 + M + UI groups clearly decreased when the TPX2 gene was silenced. The reduction in cell proliferation due to TPX2 silencing and its joint action with microvesicles and/or UI occurred in a time-dependent manner. The number of cells in the siRNA-TPX2 $+\mathrm{M}$, siRNA-TPX2 + UI, and siRNA + M + UI groups treated for 24 and $48 \mathrm{~h}$ was significantly different compared with the control $(\mathrm{P}<0.05$ or $\mathrm{P}<0.01$; Fig. 1C). The treatment time of $24 \mathrm{~h}$ was selected for further experiments.

Cell migration and invasion inhibited. Cell migration and invasion of SKVO3 cells were detected via Transwell assay. In the siRNA-TPX2 group, cell migration and invasion were decreased to $82.21 \pm 8.14 \%$ and $79.02 \pm 8.52 \%$, respectively, compared with normal SKOV3 cells. When TPX2 silencing was combined with microvesicles and ultrasonic irradiation, cell migration and invasion were further inhibited $(\mathrm{P}<0.05$ or $\mathrm{P}<0.01$ ), particularly in the siRNA-TPX $2+\mathrm{M}+\mathrm{UI}$ group, with only $41.46 \pm 4.34 \%$ of cell migration and $37.64 \pm 4.04 \%$ of cell invasion retained in comparison with the control ( $\mathrm{P}<0.01$; Fig. 2).

Expression of E-cadherin and TIMP-2 is upregulated, while that of MTA1 and MMP2 is downregulated. The production 
A
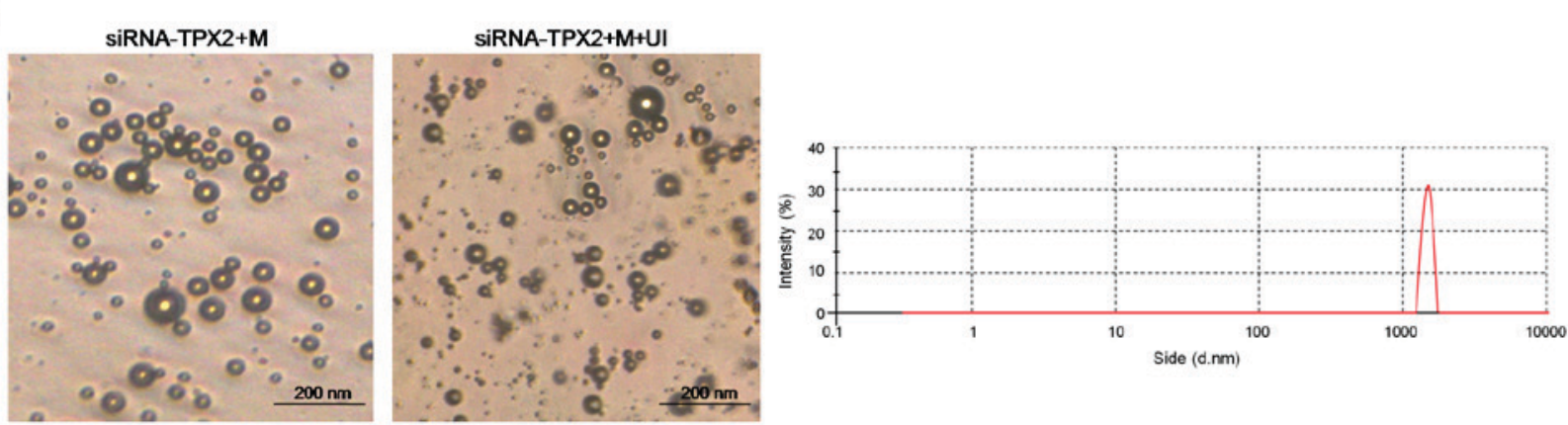

B
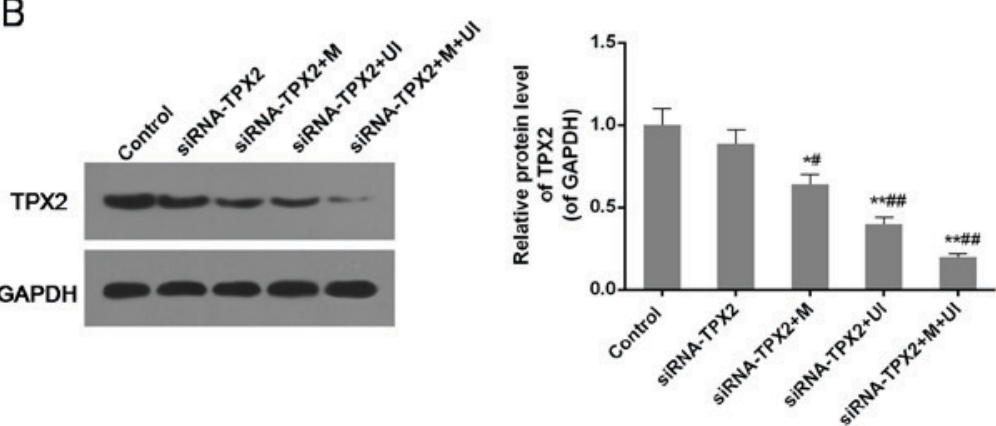

C

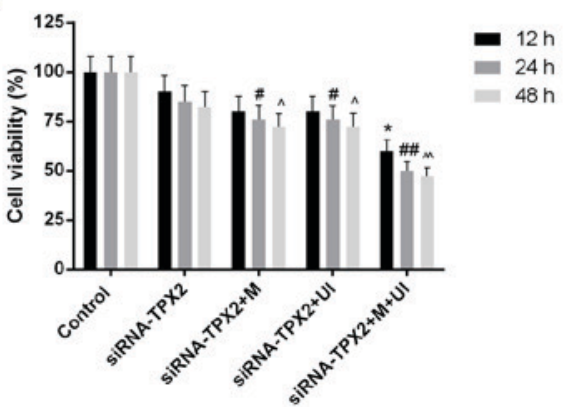

Figure 1. Identification of microvesicles, and the expression level of TPX2 and cell viability in the control group, siRNA-TPX2 group, siRNA-TPX2 + M group, siRNA-TPX2 + UI group and siRNA-TPX2 + M + UI group. (A) M were clearly identified in the siRNA-TPX2 + M group (left) and the siRNA-TPX2 + M + UI group (right), with a $1071 \pm 469.04 \mathrm{~nm}$ grain size and an $11.35 \pm 2.11 \mathrm{~mA}$ average electric potential. (B) The protein expression level of TPX2 was substantially inhibited in M-and/or UI-mediated TPX2 silencing, particularly in siRNA-TPX2 + M + UI group. Data are presented as the mean \pm standard deviation (n=3). ${ }^{*} \mathrm{P}<0.05$ and ${ }^{* *} \mathrm{P}<0.01$ vs. control; ${ }^{*} \mathrm{P}<0.05$ and ${ }^{\# /} \mathrm{P}<0.01$ vs. siRNA-TPX2. (C) Cell viability was decreased in M-and/or UI-mediated TPX2 silencing, particularly in the siRNA-TPX2 + M + UI group. Data are presented as the mean \pm standard deviation $n=3$. ${ }^{*} \mathrm{P}<0.05$ vs. control $(12 \mathrm{~h}) ;{ }^{\#} \mathrm{P}<0.05$ and ${ }^{\# \#} \mathrm{P}<0.01 \mathrm{vs}$. control $(24 \mathrm{~h}) ;{ }^{\wedge} \mathrm{P}<0.05$ and $^{\wedge \wedge} \mathrm{P}<0.01$ vs. control (48 h). siRNA, small interfering RNA; M, microvesicles; UI, ultrasonic irradiation; TPX2, targeting protein for Xklp2.

of mRNA and proteins of E-cadherin, TIMP-2, MTA1 and MMP2 from their respective genes was detected by means of RT-qPCR and western blotting (Fig. 3). These variables (the level of mRNA and proteins) for these four genes were similar (Fig. 3A-C and E). In the siRNA-TPX2 group, the genes of E-cadherin and TIMP-2 were increasingly expressed, while those for MTA1 and MMP2 were decreasingly expressed upon TPX2 silencing. The differences in expression of all the genes, however, were observed to be insignificant between treatments and control. Silencing of TPX2 in combination with microvesicles and/or ultrasonic irradiation significantly strengthened the alterations in the mRNA and protein expression levels of E-cadherin, TIMP-2, MTA1 and MMP2. In the siRNA-TPX2 + M + UI group, the expression of E-cadherin and TIMP-2 almost doubled, while that of MTA1 and MMP2 was reduced to less than one-quarter compared with the expression in siRNA-TPX2 group ( $\mathrm{P}<0.01$; Fig. 3A-E). The results of the gelatin zymography assay for MMP2 activity were consistent with the expression of MMP2 (Fig. 3F).

Phosphorylation levels of $p 38$ and JNK are restricted. The protein levels of phosphorylated p38 and JNK were observed to decrease through RT-qPCR analysis in the siRNA-TPX2 group compared with control SKOV3 cells. These levels were even lower in the siRNA-TPX2 + M, siRNA-TPX2 + UI and siRNA-TPX $2+\mathrm{M}+\mathrm{UI}$ groups $(\mathrm{P}<0.05$ or $\mathrm{P}<0.01)$, particularly when ultrasonic irradiation and microvesicles were combined to mediate the RNA silencing of TPX2 ( $<<0.01)$. p38 and JNK levels were not affected, which resulted in marked decreases in the ratios of phosphorylated to un-phosphorylated proteins in microvesicles- and/or ultrasonic irradiation-mediated TPX2 silencing, particularly in the siRNA-TPX2 $+\mathrm{M}+$ UI group $(\mathrm{P}<0.01$; Fig. 4).

\section{Discussion}

Malignant tumor cells are characterized by uncontrolled cell growth and an aberrant ability to differentiate and infiltrate the body, which enables them to cause metastasis. Cell migration and invasion are two of the primary causes of mortality in patients with cancer $(11,19)$. A number of research studies have provided evidence that TPX2 silencing may decrease cell viability and proliferation in numerous types of cancer, including colon, cervical and hepatic cancers $(12,13,15)$. It has additionally been reported that siRNA-TPX2 induces cellular apoptosis in hepatoma cells, and markedly decreases the growth and weight of already-developed xenograft tumors in nude mice; these findings suggest that a reduction in TPX2 levels may be a potential way of treating cancer $(12,17,19-21)$. Although viral vectors are known to possess high transfection efficiency, they have poor targeting ability and low security $(19,20)$. Therefore, improving the transfection efficiency of non-viral vectors is crucial to improve the curative effects of these plasmids and, thus, reduce associated complications in the treatment of cancer. 
A

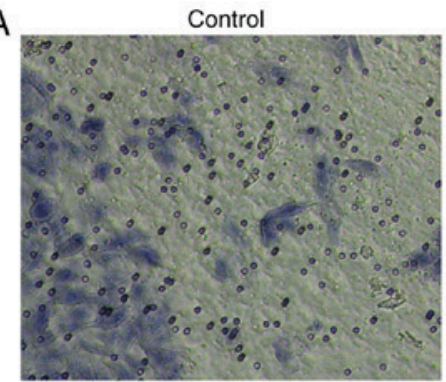

SiRNA-TPX2+UI

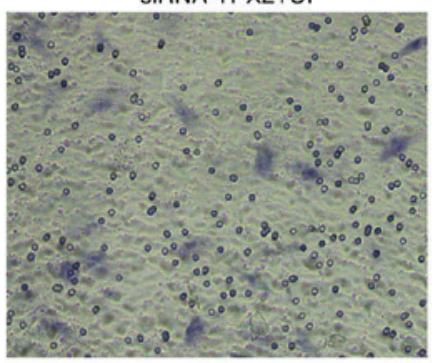

B

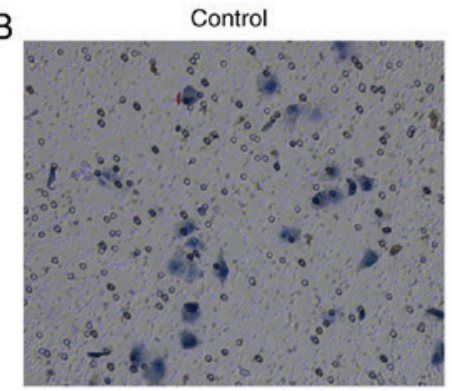

SiRNA-TPX2+UI

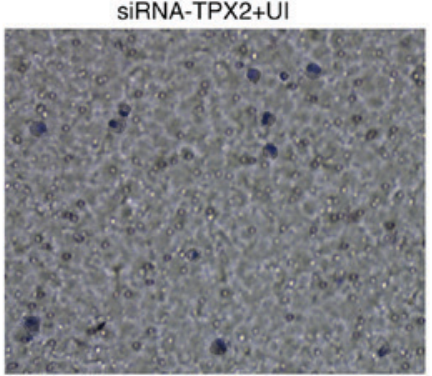

SiRNA-TPX2

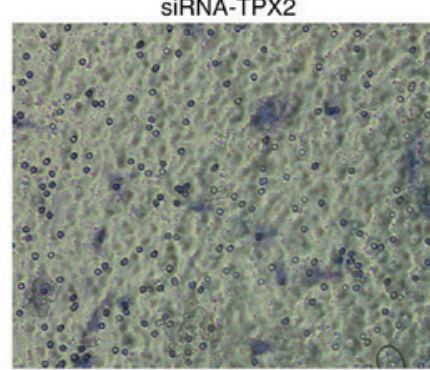

SiRNA-TPX2+M+UI

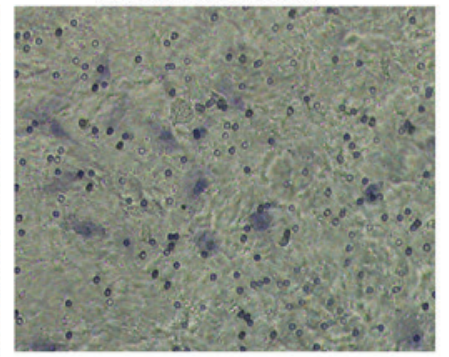

SIRNA-TPX2

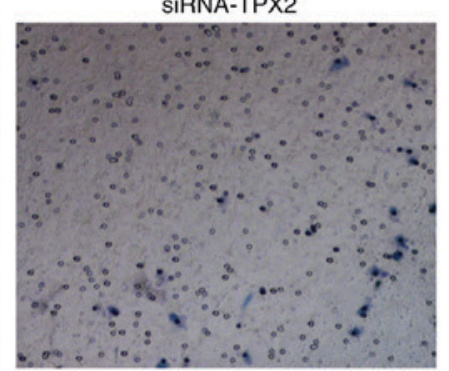

SiRNA-TPX2+M+UI

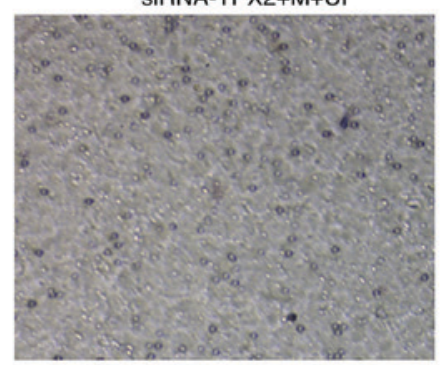

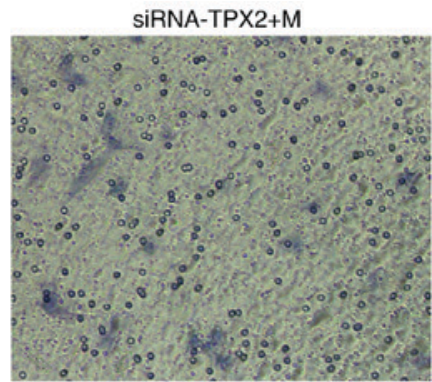

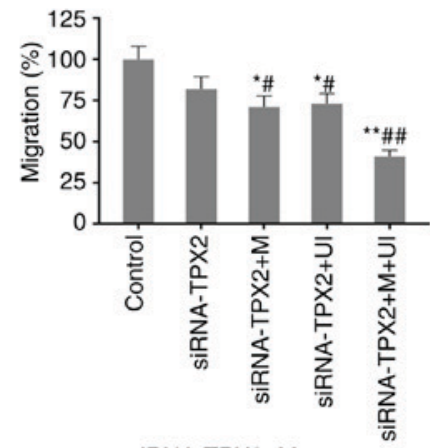

SiRNA-TPX2+M
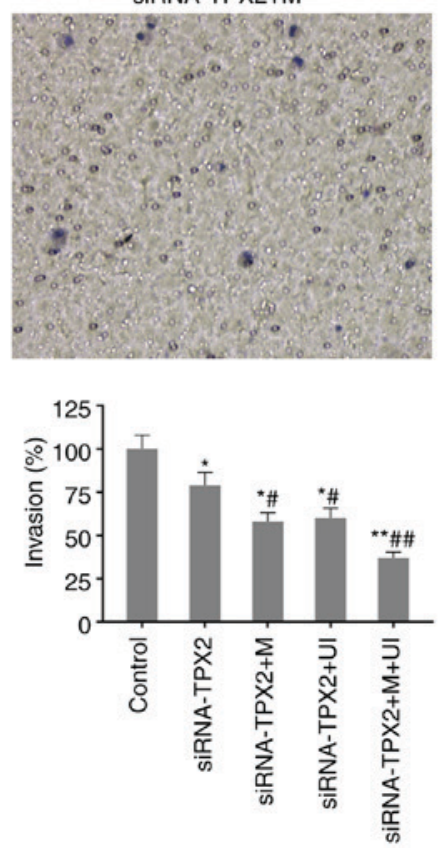

Figure 2. Migration and invasion of SKOV3 in the control group, siRNA-TPX2 group, siRNA-TPX2 + M group, siRNA-TPX2 + UI group and siRNA-TPX2 + M + UI group. (A) Cell migration was clearly inhibited in M-and/or UI-mediated TPX2 silencing, particularly in the siRNA-TPX2 + M + UI group. (B) Cell invasion was markedly inhibited in M-and/or UI-mediated TPX2 silencing, particularly in the siRNA-TPX2 + M + UI group. Invaded cells were counted under an inverted microscope (magnification, $\mathrm{x} 100$ ). Data are presented as the mean \pm standard deviation $\mathrm{n}=3$. ${ }^{*} \mathrm{P}<0.05$ and ${ }^{* *} \mathrm{P}<0.01 \mathrm{vs}$. control; ${ }^{\#} \mathrm{P}<0.05$ and ${ }^{\# \prime} \mathrm{P}<0.01$ vs. siRNA-TPX2. siRNA, small interfering RNA; M, microvesicles; UI, ultrasonic irradiation; TPX2, targeting protein for Xk1p2.

Microvesicles are a type of non-invasive vector, whose destruction by UI may generate high-energy shockwaves or microjets in the endothelial cell membrane, which subsequently induce shear stress so as to increase membrane permeability (5). The increase in permeability possibly results from the formation of temporary holes in the cell plasma or nuclear membranes. These non-lethal holes allow extracellular macromolecules and particles into cells, an effect termed sonoporation, which promotes the delivery of genes or drugs into the cells by microvesicles. Rapid shock administered to microvesicles in cell membranes facilitates the transport of DNA through the cell membranes (4). Ultrasonic waves monitor whether the microvesicle-based contrast agent has arrived at the targeting tissue in real-time; it is possible to use the wave to break up the microvesicle at a specific time and place in order to improve the curative effect (6).

The present study investigated the transfection efficiency of the plasmid siRNA-TPX2 when mediated by ultrasonic irradiation and microvesicles in SKOV3 cells, in order to evaluate its effect on growth and apoptosis in cancer cells. It was identified that conventional transfection with the siRNA-TPX2 plasmid only slightly downregulated the expression of TPX2 

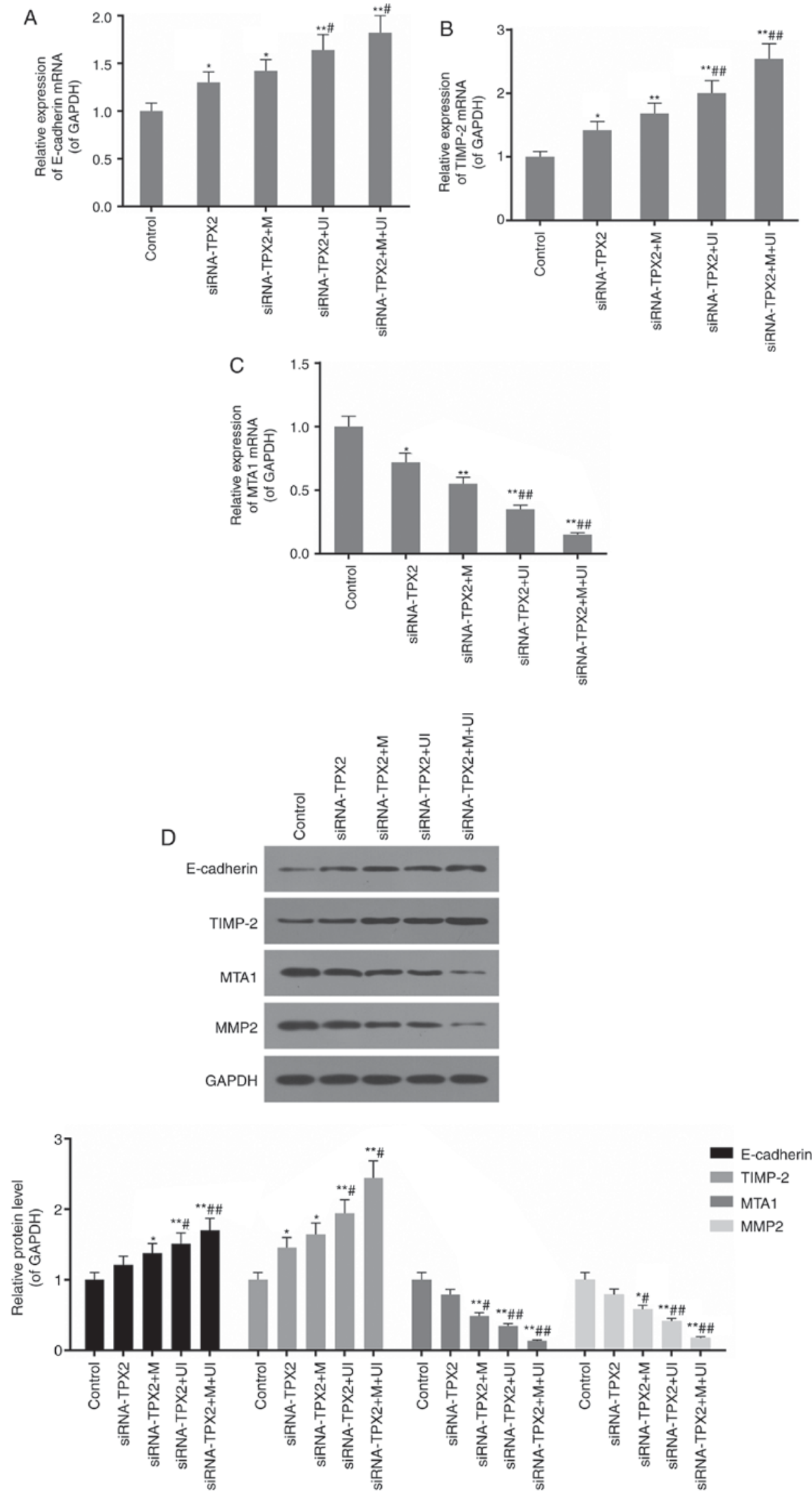

Figure 3. Expression levels of E-cadherin, TIMP-2, MTA1 and MMP2 in the control group, siRNA-TPX2 group, siRNA-TPX2 + M group, siRNA-TPX2 + UI group and siRNA-TPX $2+\mathrm{M}+$ UI group. The expression of (A) E-cadherin and (B) TIMP-2 mRNA was upregulated by M-and/or UI-mediated TPX2 silencing, particularly in the siRNA-TPX2 + M + UI group. (C) The expression of MTA1 mRNA was downregulated by M- and/or UI-mediated TPX2 silencing, particularly in the siRNA-TPX2 + M + UI group. (D) The protein expression levels of E-cadherin and TIMP-2 were upregulated, while the levels of MTA1 and MMP2 were downregulated by M-and/or UI-mediated TPX2 silencing, particularly in the siRNA-TPX2 + M + UI group. 

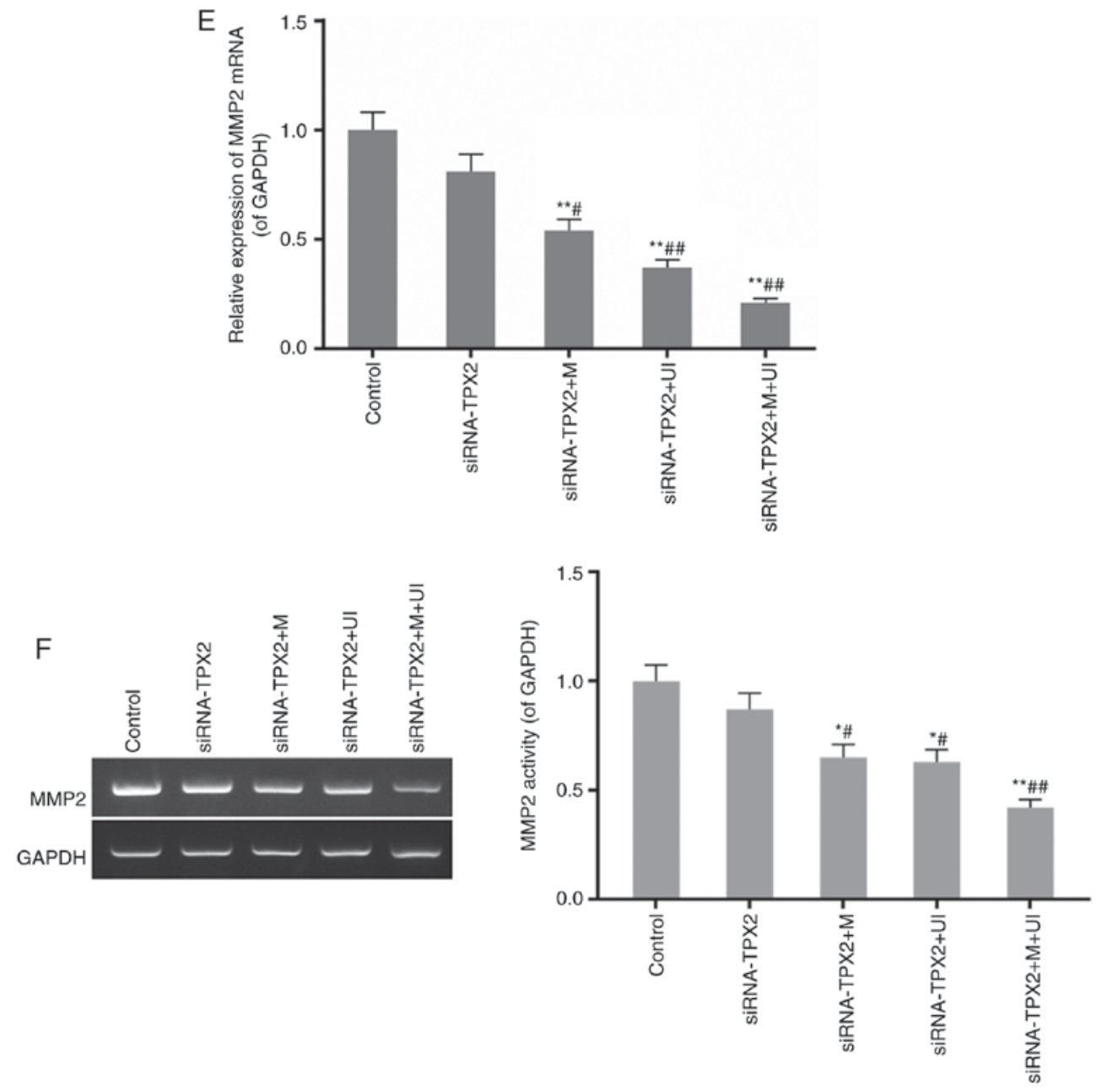

Figure 3. Continued. (E) The expression of MMP2 mRNA and (F) the activity of MMP2 were downregulated by M- and/or UI-mediated TPX2 silencing, particularly in the siRNA-TPX2 + M + UI group. Data are presented as the mean \pm standard deviation $n=3 .{ }^{*} \mathrm{P}<0.05$ and ${ }^{* * *} \mathrm{P}<0.01$ vs. control; ${ }^{*} \mathrm{P}<0.05$ and ${ }^{\# \# P} \mathrm{P}<0.01$ vs. siRNA-TPX2. E-, epithelial; TIMP-2, metalloproteinase inhibitor 2; MTA1, metastasis associated 1; MMP2, matrix metallopeptidase 2; siRNA, small interfering RNA; M, microvesicles; UI, ultrasonic irradiation; TPX2, targeting protein for Xklp2.

so as to reduce cell viability, and inhibit cell migration and invasion. The results demonstrated the inhibitory effect of TPX2 silencing on the growth of SKOV3 cells; however, the efficiency of transfection was not significant without the provision of other techniques. Introduction of microvesicles and ultrasonic irradiation was observed to markedly improve the transfection efficiency of siRNA-TPX2 into the cells, and the expressed TPX2 level was reduced to almost one-fifth of that in normal control cells. With weak expression of TPX2 in siRNA-TPX $2+\mathrm{M}+\mathrm{UI}$ group, cell viability was markedly inhibited, and cell migration and invasion were significantly reduced by over one-half in comparison with that in the siRNA-TPX2 group.

Calcium-dependent adhesions (cadherin) are a class of $\mathrm{Ca}^{2+}$-dependent transmembrane proteins, including three classical subtypes: E (epithelial) -, N (neural) -, and VE (vascular endothelial) -cadherins $(22,23)$. E-cadherins, which mediate $\mathrm{Ca}^{2+}$-dependent cell adhesion in epithelial tissues, are associated with cell recognition, differentiation, morphogenesis and tumor suppression $(24,25)$. Previous studies have identified that weak or no expression of E-cadherin is associated with metastasis and infiltration of various malignant tumors, including primary ovarian, esophagus, cervical and lung cancer $(26,27)$. MMPs and their inhibitors (TIMPs) serve important roles in extracellular matrix turnover (28). For example, MMP2, together with MMP9, is able to degrade the most plentiful component of the basement membrane, type IV collagen. Degradation of the basement membrane allows cancer cells to migrate out of the tumor, resulting in metastases. MTA1, a member of the MTA family, is an integral component of the nucleosome remodeling and histone deacetylation complexes. In the early 1990s, it was reported to be overexpressed in highly metastatic cells compared with non-metastatic cells (29). The MTA1 protein suppresses the expression of numerous tumor suppressor genes and, therefore, facilitates cell migration and invasion (30). The present study noted that TPX2 silencing slightly increased the expression of E-cadherin and TIMP2, and decreased that of MMP2 and MTA1, so as to improve cell adhesion and provide protection against membrane degradation. With the application of microvesicles and ultrasonic radiation during the process of siRNA-TPX2 transfection, the effects of TPX2 silencing on the expression of these genes were much stronger. In the siRNA-TPX2 + M + UI group, E-cadherin and TIMP-2 were more highly expressed, while MMP2 and MTA1 were more weakly expressed than 

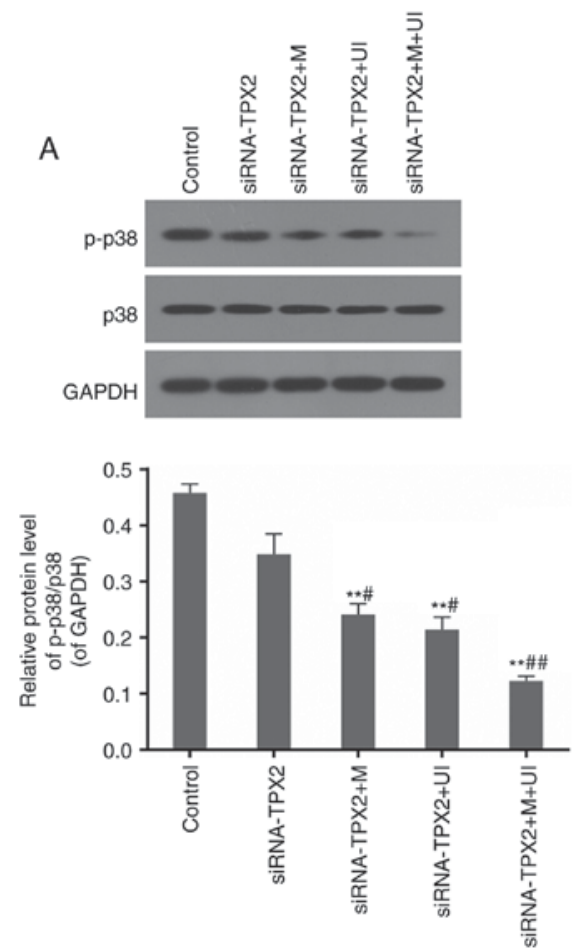
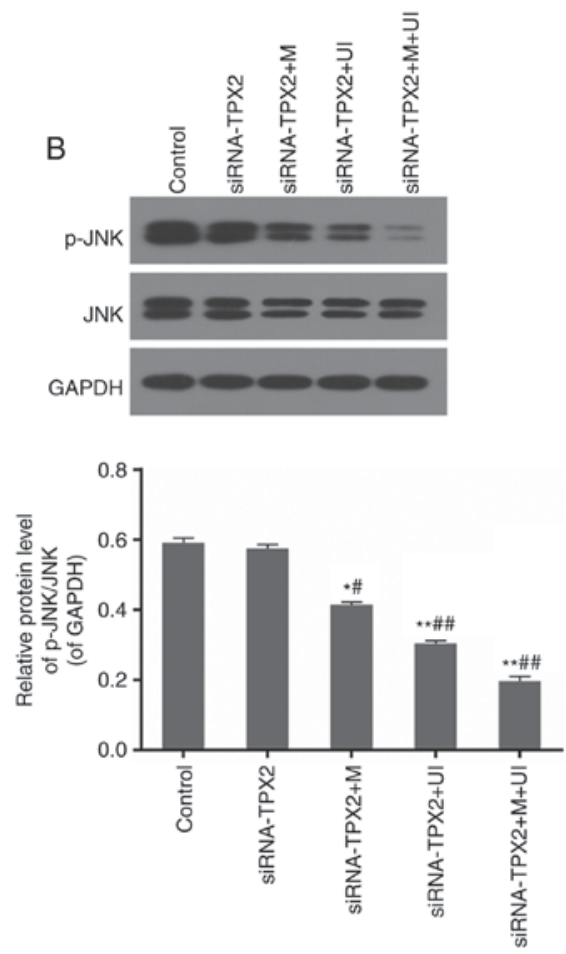

Figure 4. Protein levels of p-p38, p38, p-JNK and JNK in the control group, siRNA-TPX2 group, siRNA-TPX2 + M group, siRNA-TPX2 + UI group and siRNA-TPX2 + M + UI group. (A) The phosphorylation of p38 was inhibited by M-and/or UI-mediated TPX2 silencing, particularly in the siRNA-TPX2 + M + UI group. (B) The phosphorylation of JNK was inhibited by M- and/or UI-mediated TPX2 silencing, particularly in the siRNA-TPX2 + M + UI group. Data are presented as the mean \pm standard deviation $\mathrm{n}=3$. ${ }^{\mathrm{P}} \mathrm{P}<0.05$ and ${ }^{* *} \mathrm{P}<0.01$ vs. control; ${ }^{*} \mathrm{P}<0.05$ and ${ }^{\# \#} \mathrm{P}<0.01$ vs. siRNA-TPX2. p-, phosphorylated; JNK, c-Jun N-terminal kinase; siRNA, small interfering RNA; M, microvesicles; UI, ultrasonic irradiation; TPX2, targeting protein for Xklp2.

in other groups, which led to an improved inhibition of cell migration and invasion.

Mitogen-activated protein kinases (MAPKs), a series of serine/threonine protein kinases, are essential signaling components that convert external or internal stimuli to a cell reaction or signaling pathway. These MAPK signaling pathways make up an intricate network of signaling cascades, which regulate a variety of physiological processes, including cell migration (31). The primary function of MAPKs is the phosphorylation of phospholipases, transcription factors and cytoskeletal proteins to conduct extracellular signals into and across the cells. As two primary MAPK signal pathway members, JNKs and p38 regulate cell proliferation, differentiation and survival rates in addition to the migration of specific types of cells $(32,33)$. The results of the present study indicated a decrease in the protein expression levels of phosphorylated p38 and JNK when TPX2 was silenced, meaning that p38 and JNK pathways were restricted in M- and/or UI-mediated TPX2 silencing, particularly in the siRNA-TPX $2+\mathrm{M}+\mathrm{UI}$ group. In this group, the effect of TPX2 silencing was more pronounced compared with the other groups. The combined action of microvesicles and ultrasonic irradiation improved the transfection effect of siRNA-TPX2 to inhibit the phosphorylation of p38 and JNK.

The observations of the present study suggested that targeted inactivation of TPX2 may possess therapeutic benefits in the treatment of ovarian cancer by upregulating E-cadherin and TIMP2, and downregulating MMP2 and MTA1; it also helped inhibit the phosphorylation of p38 and JNK. The combined action of microvesicles and ultrasonic irradiation was demonstrated to markedly improve the transfection efficiency of the siRNA-TPX2 plasmid to control cell migration and invasion in ovarian cancer.

\section{Acknowledgements}

Not applicable.

\section{Funding}

No funding was received.

\section{Availability of data and materials}

The analysed data sets generated during the study are available from the corresponding author on reasonable request.

\section{Authors' contributions}

DH and MW designed the study. CY and JC performed the experiments. DH was the major contributor in the writing of the manuscript. All authors read and approved the final manuscript.

\section{Ethics approval and consent to participate}

The present study was approved by the Ethics committee of Sir Run Run Shaw Hospital, School of Medicine, Zhejiang University (Hangzhou, China). 


\section{Consent for publication}

Not applicable.

\section{Competing interests}

The authors declare that they have no competing interests.

\section{References}

1. Siegel R, Naishadham D and Jemal A: Cancer statistics, 2013 CA Cancer J Clin 63: 11-30, 2013.

2. Siegel RL, Miller KD and Jemal A: Cancer Statistics, 2017. CA Cancer J Clin 67: 7-30, 2017.

3. Yang JD, Nakamura I and Roberts LR: The tumor microenvironment in hepatocellular carcinoma: Current status and therapeutic targets. Semin Cancer Biol 21: 35-43, 2011.

4. Frenkel V, Kimmel E and Iger Y: Ultrasound-induced cavitation damage to external epithelia of fish skin. Ultrasound Med Biol 25: 1295-1303, 1999.

5. Dijkmans PA, Juffermans LJ, Musters RJ, van Wamel A, ten Cate FJ, van Gilst W, Visser CA, de Jong N and Kamp O: Microbubbles and ultrasound: from diagnosis to therapy. Eur J Echocardiogr 5: 245-256, 2004.

6. Gao ZG, Fain HD and Rapoport N: Controlled and targeted tumor chemotherapy by micellar-encapsulated drug and ultrasound. J Control Release 102: 203-222, 2005.

7. Tonon G, Wong KK, Maulik G, Brennan C, Feng B, Zhang Y, Khatry DB, Protopopov A, You MJ, Aguirre AJ, et al: High-resolution genomic profiles of human lung cancer. Proc Natl Acad Sci USA 102: 9625-9630, 2005.

8. Perez de Castro I and Malumbres M: Mitotic stress and chromosomal instability in cancer: The case for TPX2. Genes Cancer 3 : 721-730, 2012

9. Neumayer G, Belzil C, Gruss OJ and Nguyen MD: TPX2: Of spindle assembly, DNA damage response, and cancer. Cell Mol Life Sci 71: 3027-3047, 2014.

10. Wittmann T, Wilm M, Karsenti E and Vernos I: TPX2, A nove xenopus MAP involved in spindle pole organization. J Cell Biol 149: 1405-1418, 2000

11. Ma Y, Lin D, Sun W, Xiao T, Yuan J, Han N, Guo S, Feng X Su K, Mao Y, et al: Expression of targeting protein for xklp2 associated with both malignant transformation of respiratory epithelium and progression of squamous cell lung cancer. Clin Cancer Res 12: 1121-1127, 2006.

12. Wei P, Zhang N, Xu Y, Li X, Shi D, Wang Y, Li D and Cai S TPX2 is a novel prognostic marker for the growth and metastasis of colon cancer. J Transl Med 11: 313, 2013.

13. Huang Y, Guo W and Kan H: TPX2 is a prognostic marker and contributes to growth and metastasis of human hepatocellular carcinoma. Int J Mol Sci 15: 18148-18161, 2014.

14. Shigeishi H, Ohta K, Hiraoka M, Fujimoto S, Minami M, Higashikawa K and Kamata N: Expression of TPX2 in salivary gland carcinomas. Oncol Rep 21: 341-344, 2009.

15. Chang H, Wang J, Tian Y, Xu J, Gou X and Cheng J: The TPX2 gene is a promising diagnostic and therapeutic target for cervical cancer. Oncol Rep 27: 1353-1359, 2012.

16. Gruss OJ, Wittmann M, Yokoyama H, Pepperkok R, Kufer T, Sillje H, Karsenti E, Mattaj IW and Vernos I: Chromosome-induced microtubule assembly mediated by TPX2 is required for spindle formation in HeLa cells. Nat Cell Biol 4 871-879, 2002
17. Liang B, Jia C, Huang Y, He H, Li J, Liao H, Liu X, Liu X, Bai X and Yang D: TPX2 level correlates with hepatocellular carcinoma cell proliferation, apoptosis and EMT. Dig Dis Sci 60: 2360-2372, 2015

18. Livak KJ and Schmittgen TD: Analysis of relative gene expression data using real-time quantitative PCR and the 2(-Delta Delta $\mathrm{C}(\mathrm{T})$ ) method. Methods 25: 402-408, 2001

19. Warner SL, Stephens BJ, Nwokenkwo S, Hostetter G, Sugeng A Hidalgo M, Trent JM, Han H and Von Hoff DD: Validation of TPX2 as a potential therapeutic target in pancreatic cancer cells. Clin Cancer Res 15: 6519-6528, 2009.

20. Miwa T, Kokuryo T, Yokoyama Y, Yamaguchi J and Nagino M: Therapeutic potential of targeting protein for Xklp2 silencing for pancreatic cancer. Cancer Med 4: 1091-1100, 2015.

21. Satow R, Shitashige M, Kanai Y, Takeshita F, Ojima H, Jigami T, Honda K, Kosuge T, Ochiya T, Hirohashi S and Yamada T: Combined functional genome survey of therapeutic targets for hepatocellular carcinoma. Clin Cancer Res 16: 2518-2528, 2010.

22. Klezovitch $\mathrm{O}$ and Vasioukhin V: Cadherin signaling: Keeping cells in touch. F1000Res 4: 550, 2015.

23. Li L, Bennett SA and Wang L: Role of E-cadherin and other cell adhesion molecules in survival and differentiation of human pluripotent stem cells. Cell Adh Migr 6: 59-70, 2012.

24. Faleiro-Rodrigues C, Macedo-Pinto I, Pereira D and Lopes CS: Prognostic value of E-cadherin immunoexpression in patients with primary ovarian carcinomas. Ann Oncol 15: 1535-1542, 2004.

25. Oda $\mathrm{H}$ and Takeichi M: Evolution: Structural and functional diversity of cadherin at the adherens junction. J Cell Biol 193: 1137-1146, 2011

26. Carico E, Atlante M, Bucci B, Nofroni I and Vecchione A: E-cadherin and alpha-catenin expression during tumor progression of cervical carcinoma. Gynecol Oncol 80: 156-161, 2001.

27. Fernebro E, Bendahl PO, Dictor M, Persson A, Fernö M and Nilbert M: Immunohistochemical patterns in rectal cancer: Application of tissue microarray with prognostic correlations. Int J Cancer 111: 921-928, 2004.

28. Brun JL, Cortez A, Lesieur B, Uzan S, Rouzier R and Daraï E: Expression of MMP-2, -7, -9, MT1-MMP and TIMP-1 and -2 has no prognostic relevance in patients with advanced epithelial ovarian cancer. Oncol Rep 27: 1049-1057, 2012.

29. Xue Y, Wong J, Moreno GT, Young MK, Côté J and Wang W: NURD, a novel complex with both ATP-dependent chromatin-remodeling and histone deacetylase activities. Mol Cell 2: 851-861, 1998.

30. Zhang Y and Wang XF: Post-transcriptional regulation of MTA family by microRNAs in the context of cancer. Cancer Metastasis Rev 33: 1011-1016, 2014

31. Guan JL, Trevithick JE and Hynes RO: Fibronectin/integrin interaction induces tyrosine phosphorylation of a $120-\mathrm{kDa}$ protein. Cell Regul 2: 951-964, 1991.

32. Weston CR and Davis RJ: The JNK signal transduction pathway. Curr Opin Genet Dev 12: 14-21, 2002

33. Rincon M and Davis RJ: Regulation of the immune response by stress-activated protein kinases. Immunol Rev 228: 212-224, 2009.

This work is licensed under a Creative Common Attribution-NonCommercial-NoDerivatives 4.0 International (CC BY-NC-ND 4.0) License. 